\title{
Malate:Quinone Oxidoreductase and Malic Enzyme are required for the Plant Pathogen Pseudomonas syringae pv. tomato DC3000 to Utilize Malate
}

\author{
Zabrina Ebert*, Preston Jacob, Katrina Jose, Lina Fouad, Katherine Vercellino, Steven Van Dorn, Mahaa Sidiqqi, and Eve M. Mellgren \\ Department of Biology, Elmburst College, Elmburst, IL
}

Students:ebertz@net.elmburst.edu*,preston.jacob@my.rfums.org,katrina.d.jose@net.elmburst.edu,lina.a.fouad@net.elmburst.edu, kecvercell@gmail.com,vandornsa@yahoo.com,mahaa.siddiqi@net.elmburst.edu

Mentor:mellgrene@elmburst.edu*

\begin{abstract}
Pseudomonas syringae pv. tomato strain DC3000 (DC3000) is a gram-negative bacterial plant pathogen that causes disease on tomato and the model plant Arabidopsis thaliana. Interestingly, previous studies showed that malate:quinone oxidoreductase (Mqo), an enzyme in the citric acid cycle, is required for DC3000 to cause disease on these plants. In addition, growth of DC3000 lacking the mqo gene in minimal medium with malate was significantly delayed, but eventually reached wild-type levels of growth, which is similar to growth in planta. This suggests that malate may be an important carbon source for DC3000. One reason the mqo::KO bacteria may be able to reach wild-type levels of growth in culture and plants is that the DC3000 malic ensyme may be used to complete the citric acid cycle. Our research shows that a mutant strain lacking a functional mqo gene and malic ensyme gene (mqo::KO;ME::pJP) fails to grow in minimal media cultures with malate and has reduced growth in media with citrate, indicating that both Mqo and ME are required for normal growth when utilizing these carbon sources. Future studies looking at growth of this double mutant in plants will identify how important the activities of both of these genes are for DC3000 to cause disease in plants.
\end{abstract}

\section{KEY WORDS}

Malate:quinone Oxidoreductase; Malic Enzyme; MQO; Psendomonas syringae; Arabidopsis thaliana; Malate; Citrate; DC3000

\section{INTRODUCTION}

With population growth and urbanization on the rise worldwide, there is less land left for farming and a growing demand for healthy crops. Because of this, it has become increasingly important to understand how plant pathogenic bacteria such as Pseudomonas syringae cause disease in crops. Not only does this pathogen have the potential to affect crop yields, it also has similar virulence mechanisms to many human pathogens, such as Pseudomonas aeruginosa, which is an opportunistic pathogen that can be life threatening. ${ }^{1}$ By studying virulence in this model bacterium, understanding of related bacterium can be gained.

Pseudomonas syringae pv. tomato DC3000 (DC3000) is a gram-negative bacteria that causes bacterial speck disease on plants such as Arabidopsis thaliana through colonization of the apoplast. ${ }^{2,3}$ DC3000 utilizes a Type III secretion system with a syringe-like apparatus that injects virulence factors into plant cells to manipulate cellular activities and benefit the pathogen.4, 5, 6 Disease symptoms induced by DC3000 include necrotic patches or lesions and chlorosis (yellowing), which are characteristics of speck disease..$^{3,7}$

Malate:quinone oxidoreductase (Mqo) is a flavin adenine dinucleotide (FAD)-dependent membrane associated protein. ${ }^{8}$ Mqo serves two functions within most organisms. First, Mqo catalyzes the oxidation of malate to oxaloacetate in the Tricarboxylic acid cycle (TCA) allowing for the regeneration of oxaloacetate. ${ }^{8,9}$ Secondly, Mqo is found within the electron transport chain. While other enzymes of the TCA cycle utilize NAD as an electron acceptor, the oxidation of malate to oxaloacetate by Mqo donates electrons to quinones of the electron transport chain. ${ }^{9}$ After donation the quinones are required to be oxidized to recycle the enzyme for further use. ${ }^{9}$

Surprisingly, previous studies showed that Mqo is required for DC3000 to cause disease on Arabidopsis thaliana. ${ }^{10} \mathrm{When}$ the DC3000 mqo gene was replaced with an antibiotic resistance gene to create the mqo::KO strain, growth of the bacteria in plants was delayed. However, the bacteria would reach wild-type levels four days after inoculation. ${ }^{10}$ Even with the bacteria reaching wild-type levels of growth, disease symptoms in the mqo::KO infected plants never reached the severity of the plants infected with wild-type bacteria. This suggests that bacteria must reach a certain growth during infection for disease symptoms to develop to the severity of wild-type levels. ${ }^{10}$ Decreased growth and a slow recovery was also seen in culture when malate was the only carbon source, suggesting that Mqo may be needed to utilize malate during growth in the apoplast. ${ }^{10}$ The mutant grew similar to wild-type 
DC3000 in citrate and rich media. ${ }^{10}$ The ability of the mqo::KO mutant to eventually reach wild-type levels of growth in plants and in culture suggests an alternative pathway for which malate is processed in mqo::KO.

There are several possible alternative pathways for DC3000 utilization of malate without a functioning Mqo enzyme. In Corynebacterium glutamicum, mqo disruption reduces growth on various TCA cycle intermediates, but with the addition of nicotinamide, Malate dehydrogenase (Mdh) is able to maintain the conversion of malate to oxaloacetate. ${ }^{11}$ Finding both a mqo gene and $m d h$ gene in bacteria is quite common as it is not only seen in C. glutamicum but also E. coli and B. subtilis. ${ }^{12,13}$ B. subtilis is both different and unique because it lacks mqo, causing it to fully depend upon $m d h$ and the pyruvate shunt (formed by malic enzyme and pyruvate decarboxylase) to generate oxaloacetate. ${ }^{12}$ The shunt works by moving malate to pyruvate, which then cycles back through the TCA cycle with generation of oxaloacetate coming from pyruvate decarboxylase. ${ }^{2}$ However, unlike the previously mentioned bacteria, Pseudomonas aeruginosa utilizes two Malic enzymes (ME) in the absence of Mqo. The first one lacks specificity for NAD or NADP while the second one relies heavily upon NADP for the movement of electrons. This bacterium is also capable of growing aerobically with ethanol as the only carbon and energy source, utilizing a pathway not found within DC3000.8, 10

Searches of DC3000 genomic DNA indicated that the bacteria lacks an $m d h$ gene, suggesting a possible reliance on a different enzyme in mqo::KO strains. ${ }^{8,10}$ DC3000 is predicted to have a NAD-dependent malic ensyme (ME; PSPTO_3924), which has 64\% amino acid identity and 79\% similarity to the NAD-dependent malic ensyme from E. coli. Thus, we hypothesized that the DC3000 Malic enzyme was converting malate to pyruvate in the mqo::KO mutant, allowing slow growth to eventually wild-type levels. ${ }^{10}$

To test this hypothesis, a double mutant strain of DC3000 lacking both functional Mqo and ME was made and growth of this double mutant (as well as a single mutant lacking ME) was compared to wild-type DC3000 and the mqo::KO mutant in minimal media cultures. It was hypothesized disruption of just the $M E$ gene would not have a significant effect on growth of the bacteria, while the mqo::KO;ME::pJP double mutant will grow very minimally when malate is the only available carbon source. Similar to what was found with the mqo::KO mutant, it was predicted that growth on citrate would be unaffected or slightly reduced.

\section{METHODS AND PROCEDURES}

Bacterial strains, vectors, and cloning.

In order to disrupt the ME gene in wild-type DC3000 and the mqo::KO mutant strain, a suicide vector (pJP5603) was inserted into the ME gene in both strains (Figure 1). All strains and vectors used in this study are listed in Table 1. A 639 bp fragment of the ME gene was PCR amplified using Pfu Ultra II polymerase (Agilent Technologies, Santa Clara, CA) from DC3000 genomic DNA, isolated with a genomic DNA purification kit (Promega, Madison, WI). The primers used were: ME forward 5' CTATGGATCCT'TGTTCATT'TCTTACCCTGAG 3' and ME reverse 5' GCAATCTAGAACGATCCACCATGAAAATAC 3'. The PCR product was then ligated into a pCR Blunt vector (Invitrogen, Carlsbad, CA). Transformations were completed with the pME-Blunt plasmid into competent E. coli. The vector was purified with a Promega Wizard Miniprep Kit (Promega, Madison, WI). A digest of the pME-Blunt plasmid with EcoRI was performed. The $639 \mathrm{bp}$ fragment was separated from the vector via gel electrophoresis and then gel purified. Next, the ME insert was ligated into the $3.126 \mathrm{~kb}$ suicide vector pJP5603, ${ }^{14,15}$ which was also digested with EcoRI, and transformed into E. coli. The orientation of the ME gene fragment in the pJP5603 vector was tested via sequence analysis by ACGT, INC. (Wheeling, IL) to ensure that the LacZ promoter on the pJP5603 plasmid would not drive expression of the disrupted ME gene after insertion into the DC3000 genome.

A triparental mating was conducted to transfer the suicide vector with the ME insert (pME-pJP5603) into the DC3000 and mqo::KO strains using the helper strain MM294A(pRK600). ${ }^{16}$ To select for disruption of the ME gene, DC3000 with the pMEpJP5603 plasmid was grown on plates with kanamycin, and the mqo::KO mutant with the pME-pJP5603 plasmid was grown on plates with spectinomycin and kanamycin. The strains were single colony purified by streaking single colonies onto plates with the appropriate antibiotics, and repeating this process three times. Frozen stocks of each strain were created.

Wild-type DC3000, ME::pJP, mqo::KO, and mqo::KO;ME::pJP strains were routinely grown by incubation for two days at $28^{\circ} \mathrm{C}$ in King's Broth $(\mathrm{KB})$ medium $^{17}$ unless otherwise described. Antibiotics were used the following concentrations (in $\left.\mu \mathrm{g} / \mathrm{ml}\right)$ for $P$. syringae strains: rifampin, 100; kanamycin, 25; and spectinomycin, 100. Antibiotics were used at the following concentrations for E. coli strains: kanamycin, $25 \mu \mathrm{g} / \mathrm{mL}$; spectinomycin, $80 \mu \mathrm{g} / \mathrm{mL}$; and chloramphenicol, $20 \mu \mathrm{g} / \mathrm{mL}$.

PCR to confirm disruption of the ME gene.

PCR was used to confirm the disruption of the $M E$ gene by first purifying genomic DNA from DC3000, ME::pJP, mqo::KO, and mqo::KO;ME::pJP using a Wizard genomic DNA purification kit (Promega, Madison, WI). The Taq Master Mix Kit (Qiagen, Hinden, Germany) was then used to PCR amplify a region of the genomic DNA and the pJP5603 vector inserted into the genome. Using the ME::pJP confirmation (5' CATGAGCCCACATTGATCG 3') and pJP5603 reverse primers (5' 
GGCGATTAAGTTGGGTAACG 3'; Figure 1), with the following parameters: $94^{\circ} \mathrm{C}$ for 3 minutes, then cycling from $94^{\circ} \mathrm{C}$ for 30 seconds, $55^{\circ} \mathrm{C}$ for 30 seconds, and two minutes at $72^{\circ} \mathrm{C}$ for 35 cycles. A second PCR was conducted to confirm the absence of the $m q o$ gene in the $m q 0:: \mathrm{KO}$ and $m q o:: \mathrm{KO} ; M E:: \mathrm{pJP}$ strains using mqo forward primer 5' GTGACAACATGGACCTGACC 3' and mqo reverse primer 5' CTCCAGCATGATCGACACC 3', with the same cycle parameters except an extension time of one minute. PCR products were then analyzed by gel electrophoresis using 1\% agarose gel at 100 Volts for approximately 30 minutes.

Growth of DC3000 strains in minimal media cultures.

To examine the ability of the bacterial strains to grow in single carbon sources, cultures of $5 \mathrm{~mL}$ of Hoitink Sinden media (HS) ${ }^{18}$ with either $10 \mathrm{mM}$ malate or $10 \mathrm{mM}$ citrate and $\mathrm{pH}$ adjusted to 6.5 were added into sterile culture tubes. DC3000 strains were grown in $5 \mathrm{~mL} \mathrm{~KB}$ media for two days, then $3 \mathrm{~mL}$ of cells from each $\mathrm{KB}$ culture were collected and re-suspended in $1 \mathrm{~mL} 10 \mathrm{mM}$ $\mathrm{MgSO}_{4}$. Bacterial cells were washed 2 times with $10 \mathrm{mM} \mathrm{MgSO}_{4}$ and all cultures were adjusted to the same concentration $\left(\mathrm{OD}_{600}\right.$ reading between $0.1-0.2$ or $4 * 10^{7}$ cells $/ \mathrm{mL}-8 * 10^{7}$ cells $/ \mathrm{mL}$ ). $500 \mu \mathrm{L}$ of each strain was added to sterile tubes containing the $5 \mathrm{~mL}$ of HS media supplemented with malate or citrate. Three replicates of each strain were grown in minimal media with each carbon source, and the levels of growth were monitored every four hours over a 40 hour time period by measuring OD 600 absorbance with GLOMAX Multi detection system microplate reader (Promega, Madison, WI). Three control tubes of HS and malate or citrate without bacteria were also included. When analyzing the data, each sample was first blanked by subtracting the average absorbance from its corresponding media-only controls. Growth curves with $95 \%$ confidence intervals were fit for the data using local polynomial regression fitting and plotted with the ggplot2 package in $\mathrm{R}$.

\section{RESULTS}

The DC3000 strains ME::pJP and mqo::KO;ME::pJP have disrupted ME genes.

Previously, a potential malic ensyme gene (ME; PSPTO_3924) was identified in the DC3000 genome that is 1722 bp in length and has $64 \%$ amino acid identity and $79 \%$ similarity to the malic enzyme from E. coli. ${ }^{10}$ It was suggested that this Malic enzyme could convert malate to pyruvate, possibly allowing for growth of the mqo::KO mutant in minimal media containing malate. To test this hypothesis, the $M E$ gene was disrupted by inserting a suicide plasmid, which lacks an origin of replication that functions in DC 3000, ${ }^{14}$ in the middle of the ME coding sequence. This was accomplished by cloning a 639 bp section (bases 394 to 1032 ) of the ME gene into the pJP5603 vector, and introducing this plasmid into DC3000, where the plasmid integrated through homologous recombination (Figure 1). DC3000 cells that integrated the plasmid were selected by growth on kanamycin plates. The resulting strains were named $M E:: \mathrm{pJP}$ and mqo::KO;ME::pJP (Table 1).

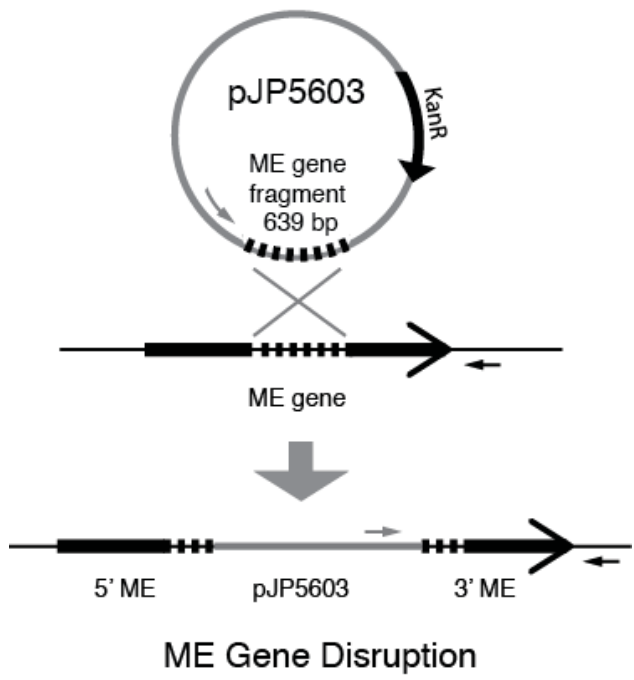

Figure 1: Distuption of the ME gene by plasmid integration into the DC3000 genome. An internal fragment of the ME gene was cloned into the pJP5603 plasmid. The new plasmid, pME-pJP5603, was introduced to DC3000 and allowed to integrate in the middle of the ME gene coding sequence. This strategy was used to create the single mutant $M E:: \mathrm{pJP}$ strain and the plasmid was introduced into the mqo::KO strain to create the double mutant mqo::KO;ME::pJP strain. Location of the primers used to confirm the disruption of the ME gene are shown by the small grey arrow (pJP5603 reverse) and small black arrow (ME::pJP confirmation). 


\begin{tabular}{|c|c|c|}
\hline Strain or Plasmid & Characteristics & Reference or Source \\
\hline \multicolumn{3}{|l|}{ Pseudomonas syringae strains } \\
\hline Pseudomonas syringae pv. tomato DC 3000 & Derivative of NCPPB1106; Rifr & 2 \\
\hline$m q o:: \mathrm{KO}$ & mqo gene replaced with $\mathrm{Sm} / \mathrm{Sp}^{\mathrm{r}}$ gene; $\mathrm{Rif}^{\mathrm{r}}, \mathrm{Sp}^{\mathrm{r}}$ & 10 \\
\hline ME::pJP & $M E$ gene disrupted with pJP5603 vector & This study \\
\hline Mqo::KO;ME::pJP & $m q o$ gene replaced and $M E$ gene disrupted & This study \\
\hline \multicolumn{3}{|l|}{ Escherichia coli strains } \\
\hline DH5-alpha Lambda-pir & recA, lacZ $\Delta M 15$, Lambda-pir & 19 \\
\hline MM294A (pRK600) & Triparental mating helper strain; $\mathrm{Cm}^{\mathrm{r}}$ & 16 \\
\hline \multicolumn{3}{|l|}{ Plasmids } \\
\hline pJP5603 & Suicide vector; $\mathrm{Km}^{\mathrm{r}}$ & 14 \\
\hline pCR Blunt & Cloning vector; $\mathrm{Km}^{\mathrm{r}}$ & Invitrogen \\
\hline $\mathrm{p} M E$-Blunt & pCR Blunt with ME gene fragment & This study \\
\hline $\mathrm{p} M E-\mathrm{pJP} 5603$ & pJP5603 with ME gene fragment & This study \\
\hline
\end{tabular}

Table 1: Bacterial strains and vectors used in this study.

To confirm that the $M E$ gene was disrupted in both of these strains, primers were designed that would amplify an approximately 900-1500 bp piece of DNA if the pJP5603 plasmid inserted into the ME gene, depending on exactly where the homologous recombination event occurred (see Figure 1). It was expected that the bacterial strains whose $M E$ gene was interrupted with a kanamycin resistance gene (ME:::pJP and mqo::KO;ME::pJP) would produce a PCR product, whereas DC3000 and mqo::KO would not. As seen in the Figure 2 Row 2, only ME::pJP (lane 1) and mqo::KO;ME::pJP (lane 4) show a PCR product just below $1.5 \mathrm{~kb}$, verifying malic ensyme gene disruption in these strains.

To confirm that the mqo gene was absent from the mqo::KO and mqo::KO;ME::pJP double mutant strains, PCR reactions with primers that amplify within the mqo coding sequence were used. The bacterial strains whose mqo gene was intact (DC3000 and ME:::pJP) produced a $275 \mathrm{bp}$ PCR product, whereas the mqo::KO and mqo::KO;ME::pJP strains did not have an amplified product (Figure 2, Row 1).

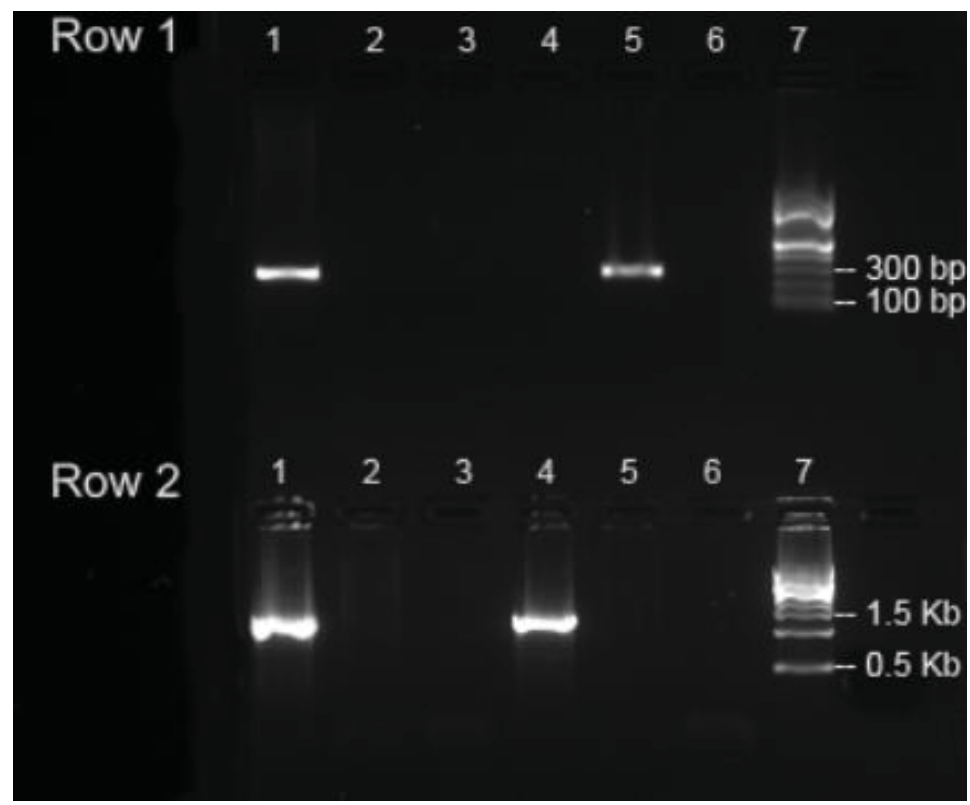

Figure 2: PCR confirmation of ME gene disruption. Row 1: Lane 7 contains the NEB 100 bp ladder. Band sizes for the 100 bp DNA ladder from top to bottom are: 1,517 bp, 1,200 bp, 1,000 bp, 900 bp, 800 bp, 700 bp, 600 bp, 500 bp, 400 bp, 300 bp, 200 bp and 100 bp. Lanes 1 through 6 contain PCR products from amplification with the mqo RT forward + mqo RT reverse primers for the following strains: lane 1 - ME::pJP, lane 2 - mqo::KO sample 1 , lane 3 - mqo::KO sample 2, lane 4 - mqo::KO;ME::pJP, lane 5 - DC3000, lane 6 - no DNA control reaction. Lanes 1 and 5 show amplification of a band of approximately 275 bp. Row 2: lane 7 contains the NEB $1 \mathrm{~Kb}$ ladder. Band sizes for the $1 \mathrm{~Kb}$ DNA ladder from top to bottom are: $10.0 \mathrm{~kb}, 8.0 \mathrm{~kb}, 6.0 \mathrm{~kb}, 5.0 \mathrm{~kb}, 4.0 \mathrm{~kb}, 3.0 \mathrm{~kb}, 2.0 \mathrm{~kb}$, $1.5 \mathrm{~kb}, 1.0 \mathrm{~kb}$ and $0.5 \mathrm{~kb}$. Lanes 1 through 6 contain PCR products from amplification with the ME::pJP confirmation + pJP5603 reverse primers for the following strains: lane 1 - ME::pJP, lane 2- mqo::KO sample 1, lane 3 - mqo::KO sample 2, lane 4 - mqo::KO;ME::pJP, lane 5 - DC3000, lane 6 - no DNA control reaction. Lanes 1 and 4 show amplification of a band just below the $1.5 \mathrm{~kb}$ band. 
ME allows growth of the mqo::KO mutant in media containing malate.

Previous studies indicated that the mqo gene is required for wild-type growth and disease in DC3000. A knockout of the mqo gene with a spectinomycin resistance gene $(m q o:: \mathrm{KO})$ resulted in delayed growth in minimal media with malate, however, eventually wild-type growth levels were reached. ${ }^{10}$ The ability for $m q 0:: \mathrm{KO}$ to reach wild-type levels could be due to the activity of $\mathrm{ME}$, which converts malate to pyruvate. Therefore, the $M E:: \mathrm{pJP}$ single mutant and mqo::KO;ME::pJP double mutant strain were created and grown in minimal media with malate to determine the significance of the $M E$ and mqo genes in DC3000. Growth of all four strains (DC3000, mqo::KO, ME::pJP, and mqo::KO;ME::pJP) in HS minimal media cultures with malate (Figure 3A) showed, as expected, delayed growth of mqo::KO, which reached wild-type levels by the end of 40 hours. The ME::pJP strain grew similar to DC3000 throughout the 40 hour period, but did show a slightly longer lag phase in some experiments (not shown). The mqo::KO;ME::pJP double mutant shows little to no growth throughout the curve (Figure 3A). Failure of mqo::KO;ME::pJP to grow in malate indicates the double mutant's inability to utilize malate as a carbon source.

A

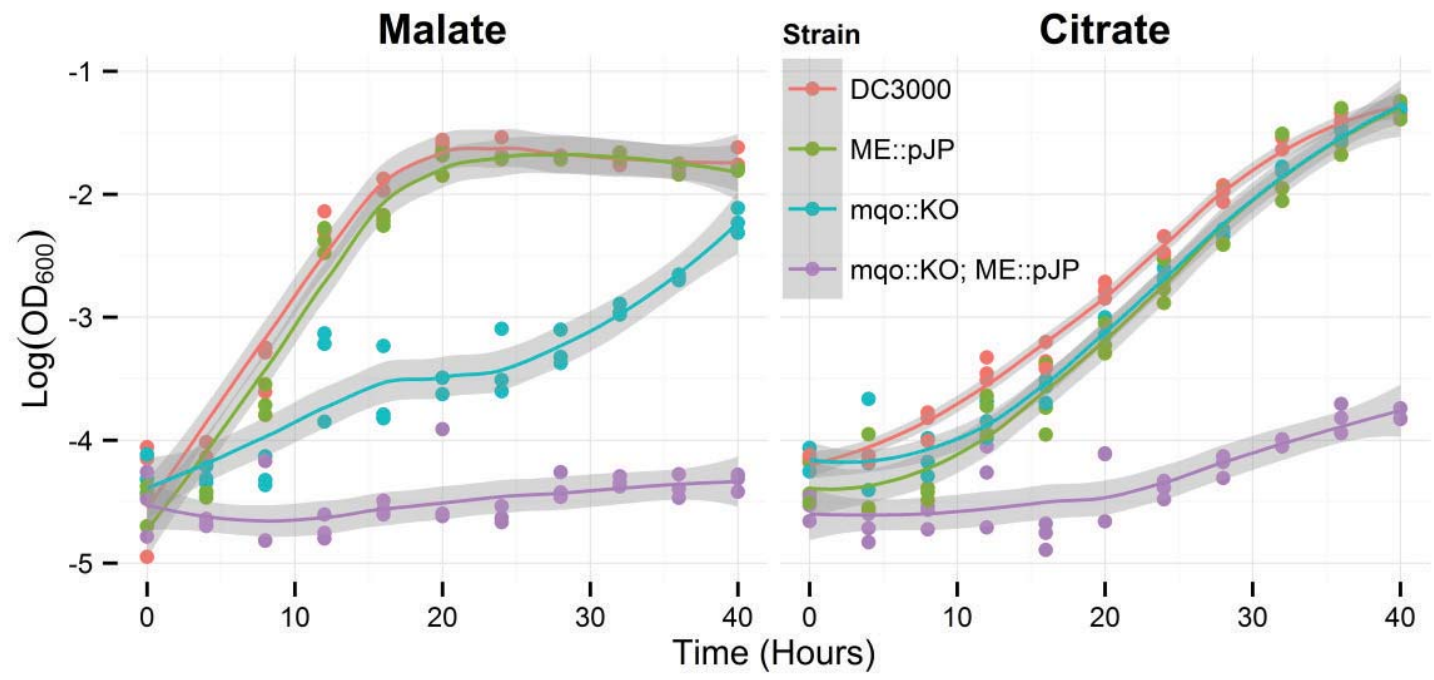

Figure 3: Growth of DC3000, ME::pJP, mqo::KO, and mqo::KO;ME::pJP in HS minimal media with 10mM malate (A) or citrate (B). Each point represents an individual culture absorbance reading and the shaded area around the curves represents the 95\% confidence interval. Each growth curve was replicated at least three times with similar results.

$M q o$ and $M E$ are required for growth in minimal media with citrate.

In previous studies, ${ }^{10}$ the mqo::KO mutant did not show a growth defect when growing in HS minimal media cultures with citrate. Thus, it was hypothesized that growth of the double mutant in minimal media with citrate as its sole carbon source would generate a sufficient amount of ATP (which is generated by intermediates in the TCA cycle) to facilitate growth. When comparing growth in HS minimal media with citrate, (Figure 3B) both the mqo::KO and the ME::pJP had growth rates similar to wild-type bacteria for the entire 40 hour time period. In comparison, the mqo::KO;ME::pJP strain had a much reduced growth rate throughout the curve (Figure 3B). This indicates that in the absence of Mqo, ME is required for wild-type growth when citrate is the only available carbon source.

\section{DISCUSSION AND CONCLUSIONS}

Previous work showed that the mqo gene is required for virulence of the plant pathogen DC3000. However, a remaining question was why the mqo::KO mutant was able to grow in minimal media cultures with malate, despite lacking the enzyme for the last step in the TCA cycle where malate is converted to oxaloacetate. It was hypothesized that activity of the ME gene was allowing DC3000 to grow when Mqo is inactive. To answer this question, a mutant strain lacking mqo and with a disrupted $M E$ gene was created. Our current work supports the hypothesis, as we show that when the mqo gene is absent and the ME gene is disrupted, DC3000 can no longer grow in minimal media with malate.

The results obtained from this study show that the $M E$ gene is required for utilization of malate by DC3000 in the absence of $m q o$. The fact that the mqo::KO mutant has a more pronounced growth defect than the ME single mutant suggests that mqo is the primary enzyme responsible for malate utilization. Future studies could look at regulation of these genes to see if they are both used during normal growth, or if $M E$ expression is upregulated when mqo is missing. 
The mqo::KO mutant grew similarly to wild-type DC3000 in citrate, indicating that mqo was not required for normal growth when citrate is the provided carbon source. We found that the double mutant lacking mqo and a functional ME gene does not grow like wild-type DC3000. It was hypothesized that mqo::KO;ME::pJP would be able grow normally on citrate because growth in this source would allow for all of the steps of the TCA cycle before malate oxidation to be completed, reducing all electron carriers except for the final NADH in the last step of the cycle. However, the data collected (Figure 3B) shows that mqo::KO;ME::pJP has delayed growth when citrate is the available carbon source, unlike the wild-type bacteria.

One reason why the double mutant may not be able to grow like wild-type DC3000 in citrate is because of the lack of oxaloactetate. Oxaloacetate is an intermediate for synthesis of some amino acids; thus, the double mutant may be lacking a way to produce these amino acids, inhibiting growth. Alternatively, growth may be inhibited because of accumulation of an intermediate such as malate or one of the other dicarboxylates. Buildup of one of the TCA intermediates may be a signal to stop expressing some TCA cycle enzymes. Future experiments will try to distinguish between these two hypotheses by growing $m q \circ:: \mathrm{KO}$; $M E:: \mathrm{pJP}$ in HS media with citrate and supplementing with oxaloacetate.

Future experiments will also include looking at if mqo and $M E$ are important for growth in planta. We predict that the double mutant will not grow as well as the mqo::KO bacteria and will not cause any disease symptoms on infected plants. These experiments looking at the growth of the mqo::KO;ME::pJP double mutant in plants will help to confirm that malate, which is present in the plant apoplast, ${ }^{20}$ is an important carbon source for DC3000 when it is growing in this environment.

\section{ACKNOWLEDGEMENTS}

The authors thank Dr. Keith Hultman for his help with generating figures for this manuscript. This work was supported by an Elmhurst College Faculty Research Grant to Eve Mellgren and an Elmhurst College Swords Scholar Grant to Zabrina Ebert.

\section{LITERATURE CITED}

1. Hauser, A.R. (2009) The type III secretion system of Pseudomonas aeruginosa: infection by injection, Nature Reviews Microbiology 7(9), 654-665.

2. Cuppels, D. A. (1986) Generation and characterization of Tn 5 insertion mutations in Pseudomonas syringae pv. tomato, Appl Environ Microbiol 51(2), 323-327.

3. Preston, G. M. (2000) Pseudomonas syringae pv. tomato: the right pathogen, of the right plant, at the right time, Mol Plant Pathol, 1(5), 263-275.

4. Galan, J. E., and Collmer, A. (1999) Type III secretion machines: bacterial devices for protein delivery into host cells, Science 284(5418), 1322-1328.

5. Grant, S. R., Fisher, E.H., Chang, J.H. Mole, B.M., and Dangl, J. L. (2006) Subterfuge and manipulation: type III effector proteins of phytopathogenic bacteria, Annu Rev Microbiol 60, 425-449.

6. Kunkel, B.N., and Chen, Z. (2006) Virulence strategies of plant pathogenic bacteria, in The prokaryotes (Dworkin et al., Ed.) vol. 2, 421-440, Springer, New York, NY.

7. Whalen, M. C., Innes, R.W., Bent, A.F., and Staskawicz, B. J. (1991) Identification of Pseudomonas syringae pathogens of Arabidopsis and a bacterial locus determining avirulence on both Arabidopsis and soybean, The Plant Cell 3(1), 49-59.

8. Kretzschmar U., Ruckert, A., Jeoung, J., and Görisch, H. (2002) Malate:quinone oxidoreductase is essential for growth on ethanol or acetate in Pseudomonas aeruginosa, Microbiology 148(12), 3839-3847.

9. Cohn, D. V. (1956) The oxidation of malic acid by Micrococcus lysodeikticus, J Biol Chem 221, 413-423.

10. Mellgren, E.M., Kloek, A. P., and Kunkel, B. N. (2009) Mqo, a tricarboxylic acid cycle enzyme, is required for virulence of Pseudomonas syringae pv. tomato strain DC3000 on Arabidopsis thaliana, J Bacteriol 191(9), 3132-3141.

11. Molenaar, D., Van Der Rest, M. E., Drysch, A., and Yucel, R. (2000) Functions of the membrane-associated and cytoplasmic malate dehydrogenases in the citric acid cycle of Corynebacterium glutamicum, J Bacteriol 182(24), 6884-6891.

12. Ohshima, T. and Tanaka, S. (1993) Dye-linked L-malate dehydrogenase from thermophilic Bacillus species DSM 465, Eur J Biochem 214(1), 37-42.

13. Van Der Rest, M. E., Frank, C., and Molenaar, D. (2000) Functions of the membrane-associated and cytoplasmic malate dehydrogenases in the citric acid cycle of Eschericia coli, J Bacteriol 182(24), 6892-6899.

14. Penfold, R.J., Pemberton, J.M. (1992) An improved suicide vector for construction of chromosomal insertion mutations in bacteria, Gene 118(1), 145-146.

15. Riedel, T., Rohlfs, M., Buchholz, I., Wagner-Döbler, I., Reck, M. (2013) Complete sequence of the suicide vector pJP5603, Plasmid 69(1), 104-107.

16. Finan, T. M.,Kunkel, B.N., De Vos, G.F., and Signer, E.R. (1986) Second symbiotic megaplasmid in Rhizobium meliloti carrying exopolysaccharide and thiamine synthesis genes, J Bacteriol 167(1), 66-72.

17. King, E. O., Ward, M.K., and Raney, D.E. (1954) Two simple media for the demonstration of pyocyanin and fluorescein, $J$ Lab Clin Med 44(2), 301-307. 
18. Sreedharan, A., Penaloza-Vazquez, A., Kunkel, B.N., and Bender, C.L. (2006) CorR regulates multiple components of virulence in Pseudomonas syringae pv. tomato DC3000, Mol Plant-Microbe Interact 19(7), 768-779.

19. Miller, V. L., and Mekalanos, J.J. (1988) A novel suicide vector and its use in construction of insertion mutations: osmoregulation of outer membrane proteins and virulence determinants in Vibrio cholerae requires toxR, $J$ Bacteriol 170(6), 2575-2583.

20. Lee, M., Choi, Y., Burla, B., Kim, Y.Y., Jeon, B., Maeshima, M., Yoo, J.Y., Martinoia, E., and Lee, Y. (2008) The ABC transporter AtABCB14 is a malate importer and modulates stomatal response to CO2, Nat Cell Biol 10(10), 1217-1223.

\section{ABOUT THE STUDENT AUTHORS}

Zabrina Ebert is a recent graduate of Elmhurst College with a Bachelor of Science in Biology. She has worked with Dr. Eve Mellgren on Psuedomonas syringae for two years and has presented research at NCUR and NCHC. She will be attending Midwestern University in the fall of 2015 to pursue a degree in Osteopathic Medicine.

Preston Jacob graduated from Elmhurst College in 2013 with a Bachelor of Science in Biology. Preston currently attends Chicago Medical School at Rosalind Franklin University of Medicine and Sciences in North Chicago, IL where he is pursuing a degree in Allopathic Medicine.

Katrina Jose worked on this project her senior year at Elmhurst College. She is a recent graduate of Elmhurst College with a Bachelor of Arts in Biology with a minor in Psychology. She is a member of the Beta Beta Beta National Biological Honor Society, Pi Gamma Mu International Honor Society in Social Science and the American Society for Clinical Pathology. She is currently pursuing a career in Medical Laboratory Science.

Lina Fouad is a senior at Elmhurst College pursuing a Bachelor of Science in biology and psychology. She has been working on this project since spring semester of her junior year.

Katherine Vercellino graduated from Elmhurst College in May 2014 with a Bachelor of Science in Biology. She is currently working as a research scientist for Archer Daniels Midland Company.

Steven Van Dorn graduated from Elmhurst College in May 2014. He has acquired licensing as an EMT in the state of Illinois, and is considering pursuing a Master's degree in genetics or attending medical school.

Mahaa Siddiqi is a senior at Elmhurst College and is working towards her Bachelor's degree in Biology with minors in Chemistry and Medical Humanities. She is the Vice President of the pre-health national honor society Alpha Epsilon Delta as well as a national member of Beta Beta Beta. Her future plans include medical school with a specialty in infectious disease.

\section{PRESS SUMMARY}

Pseudomonas syringae pv. tomato strain DC3000 (DC3000) is a bacterial plant pathogen known to cause disease in Arabidopsis thaliana and tomato plants. Interestingly, previous studies have shown that malate:quinone oxidoreductase (Mqo), an enzyme in the tricarboxylic acid cycle, is required for DC3000 to cause disease. A mutant strain lacking the mqo gene had significantly delayed growth when malate was the only carbon and energy source, but eventually reached wild-type levels of growth. This may be due to the activation of a secondary pathway mediated by Malic Enzyme (ME). Our research shows that a mutant strain lacking both the mqo gene and the malic ensyme gene fails to grow in minimal media cultures with malate. The roles of both genes on DC3000 energy and amino acid production, as well as virulence, are the topics of future research. 